Téoros

Revue de recherche en tourisme

\title{
La signalisation touristique : une priorité pour Montréal
}

\section{Jean-Marc Bissonnette}

Volume 10, numéro 3, novembre 1991

Destination Montréal : d’hier à demain

URI : https://id.erudit.org/iderudit/1079199ar

DOI : https://doi.org/10.7202/1079199ar

Aller au sommaire du numéro

Éditeur(s)

Université du Québec à Montréal

ISSN

0712-8657 (imprimé)

1923-2705 (numérique)

Découvrir la revue

Citer cet article

Bissonnette, J.-M. (1991). La signalisation touristique : une priorité pour Montréal. Téoros, 10(3), 30-32. https://doi.org/10.7202/1079199ar d'utilisation que vous pouvez consulter en ligne.

https://apropos.erudit.org/fr/usagers/politique-dutilisation/ 


\section{Jean-Marc Bissonnette*}

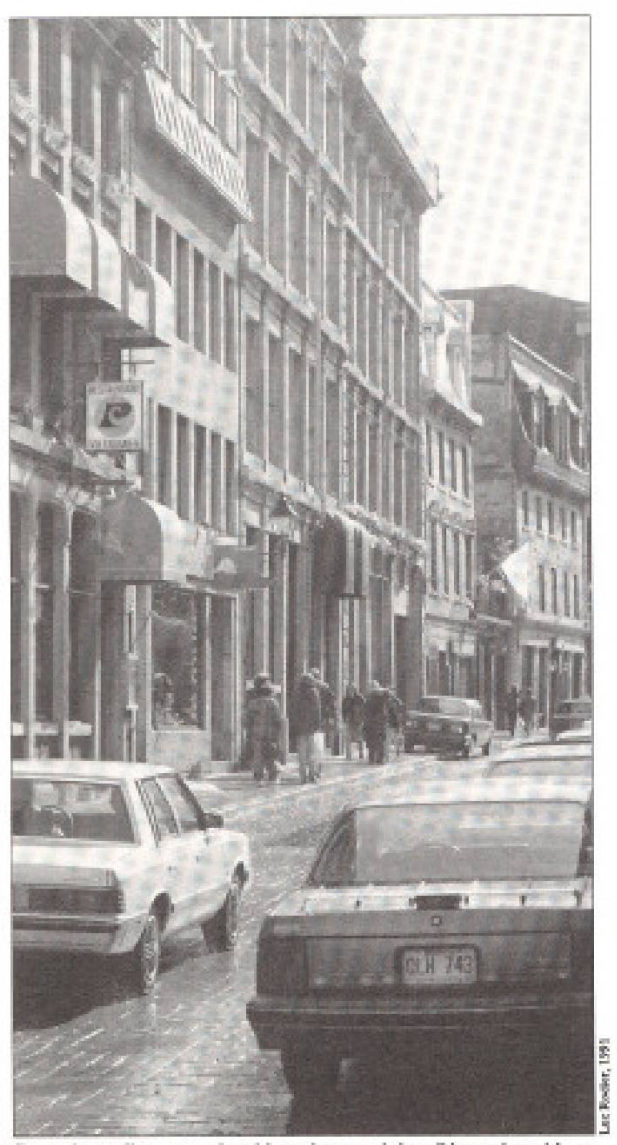

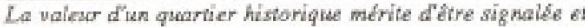
interprise,

La notion d'accueil touristique, pris dans son sens large, englobe une série de moyens visant à rendre le séjour du visiteur le plus agréable possible. À cet effet, dans le dernier numéro de Teoros, qui traitait de l'accueil touristique, monsieur Stanley Tremblay, président d'Alpha 90, soulignait que acomme la qualité des prestations de différents intervenants contribue à la qualité de l'expérience touristique du visiteur, les fonctions d'accueil prennent l'avantscène lors de la réalisation du séjours ${ }^{\text {th }}$.

Suivant cette constatation, on reconnaît d'emblée que ces fonctions, même si de l'avis de monsieur Tremblay, elles ne sont

Monsieur Jean-Marc Bissonnette est commissaireacjoint a la CIDEM-Ville de Montréal.

\section{La signalisation touristique: une priorité pour Montréal}

pas toujours clairement definies, auront une forte influence sur l'impression laissee au touriste lors de sa visite et sur l'appréciation qu'il diffusera de la qualité de son séjour.

Si l'on compte qu'une forte proportion de touristes qui viennentà Montréal le font par voie routière, la signalisation touristique de première ligne demeure, sans contredit, un des aspects les plus importants et des plus visibles de la fonction d'accueil.

A ce titre, Montréal accueilleraen 1992, de façon générale et plus particulièrement sur son réseau routier, un plus grand nombre de touristes qui y sejourneront dans le cadre

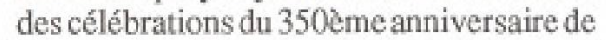
sa fondation. Les sites à visiter seront nombreux et les touristes devront se déplacer fréquemment pour assister aux manifestations. Il conviendra donc de leur offrir une signalisation qui les aidera à découvrir les attraits déjà connus de Montréal et ceux qui seront inaugurés pour l'occasion.

Sans fausse pudeur, on peut affirmer que Montréal souffre de déficiences majeures en regard de son système de signalisation touristique et que le momentum cré́ par le $350 \mathrm{eme}$ anniversaire nous donne l'occasion d'améliorer, sur une base permanente, cette facette de l'accueil, et ce, afin d'accroitre l'accessibilité au produit touristique.

Le printemps de cette année de rẹ́jouissance a donc été choisi, par les autorités de la Ville de Montréal, pour implanter le maximum d'éléments de ce nouveau système de signalisation touristique. La Ville désire, de ce fait, disposer d'une signalisation touristique adaptée à ses particularités et qui soit aussi à la fois intégrée et efficace. Cette nouvelle politique de signalisation trouve son point de départ dans la désignation de 5 concentrations d'altraits, ou pôles touristiques. Ce système comprendra essentiellement deux composantes: une signalisation routière à partir des portes d'entrée de la ville jusqu'aux stationnements d'accueil des attraits majeurs et une signalisationpiétonne quiprendra en charge le piéton pour l'aider dans son cheminement à l'intéricur des pôles touristiques.

Le Comité exécutif de la Ville de Montréal entérinait, au mois de juin 1991, l'ensemble des $́$ lements de la politique de signalisation touristique pour Montréal. Nous tenterons, dans les prochaines lignes, de présenter succinctement les travaux de recherche et de consultation mis de l'avant pour en arriver à mettre en place ce nouveau système de signalisation touristique.

\section{Les démarches}

La mise en place de cet ambitieux projet trouve sa source par l'instauration en 1988 $\mathrm{d}^{4}$ une toute nouvelle politique de signalisation touristique du gouvernement đu Qué bec, élaborée par les ministères des Transports et du Tourisme. Cette politique excluait les agglomérations de 50000 habitants et plus à qui il revenait d'implanter leur propre signalisation touristique.

Cependant, afin de suppléer à la signalisation touristique dans ces agglomérations, la politique du gouvernement prêvoyait le renforcement de la signalisation des bureaux d'information touristique pour guider les touristes vers les equipements touristiques de ces agglomérations.

La Ville de Montréal confiait, en 1988, à la firmeSÉROMmarketing le mandatde tracer le portrait de l'environnement signalétique de Montréal et de regarder son interaction avec le touriste, dans le but visé d'élaborer un scénario d'implantation d'un systeme de signalisation touristique.

Les conclusions de l'étude SÉROM(2) mettaient en relief le caractère non intégré et sans normalisation de la signalisation touristique de Montréal. En effet, le touriste qui sillonne les rues de la ville peut y rencontrer $l^{*} u n$ des quelques 60 systèmes de signalisation touristique différents qui n'ont virtuellement aucune cohérence les uns avec les autres. C'est ainsi que les routes du territoire montréalais peuvent être parfois pavoisces de panneaux bruns et blancs, indiquant des lieux tels que 
l'Oratoire St-Joseph et le centre-ville, A d'autres occasions, on peut aussi y rencontrer des panneaux blancs et verts qui signalisent des lieux de nature trè̀s différents qui vont de l'aréna à la signalisation d'une rue, telle la rue Viger. En d'autres endroits, les panneaux rouges, verts et blancs sont à l'honneur, servant à signaliser le Stade Olympique et le Jardin Botanique et qui laissent, à l'occasion, le visiteur pantois en raison de cheminements incomplets. Il en va de même en regard du Parc-des-Iles et du Vieux-Montréal, qui disposenteux aussi de systèmes multiples, dans bien des cas incomplets. Ce melting pot de systèmes de signalisation à la pièce a pour effet de ne satisfaire qu'en partie, comme le rappelle SÉROM, aux exigences de visibilité, de communication et de direction efficace des touristes.

Ils sont généralement conçus pour un système unique, sans coordination avec les autres systèmes de signalisation implantés sur le territoire. Chacun dessystèmes adopte ses propres normes et coutumes et fait en sorte que le touriste n'a pas de référend général.

Force est de constater que la multiplication des systèmes de signalisation touristique à Montréal a un effet négatif sur la qualité de l'accueil des visiteurs. Le manque de cohếrence du système dans sa globalitế et le peu d'information donnée au touriste, à partir des portes d'entrée de la ville, laisse le visiteur avec une première impression nẹgative.

Ces constatations amenèrent le consultant à se pencher sur deux prémisses de base pour l'élaboration d'un scénario de signalisation qui soit viable pour Montréal; il s'agissait évidemment de la rationalisation et de la normalisation du système de signalisation touristique. L'idée maitresse qui devait sous-tendre à l'élaboration de ce scénario visait à favoriser l'accessibilité au produit touristique sans perdre de vue que l'action se situait dans le contexte d'une grande ville.

\section{Le scénario proposé}

Dans son étude, le consultant évalua les différents scénarios autour desquels pouvait s"articuler la signalisation touristique de Montréal. Des divers modèles étudiés, le choix s'est arrêté sur la signalisation ayant comme points de départ des concentrations d'attraits.

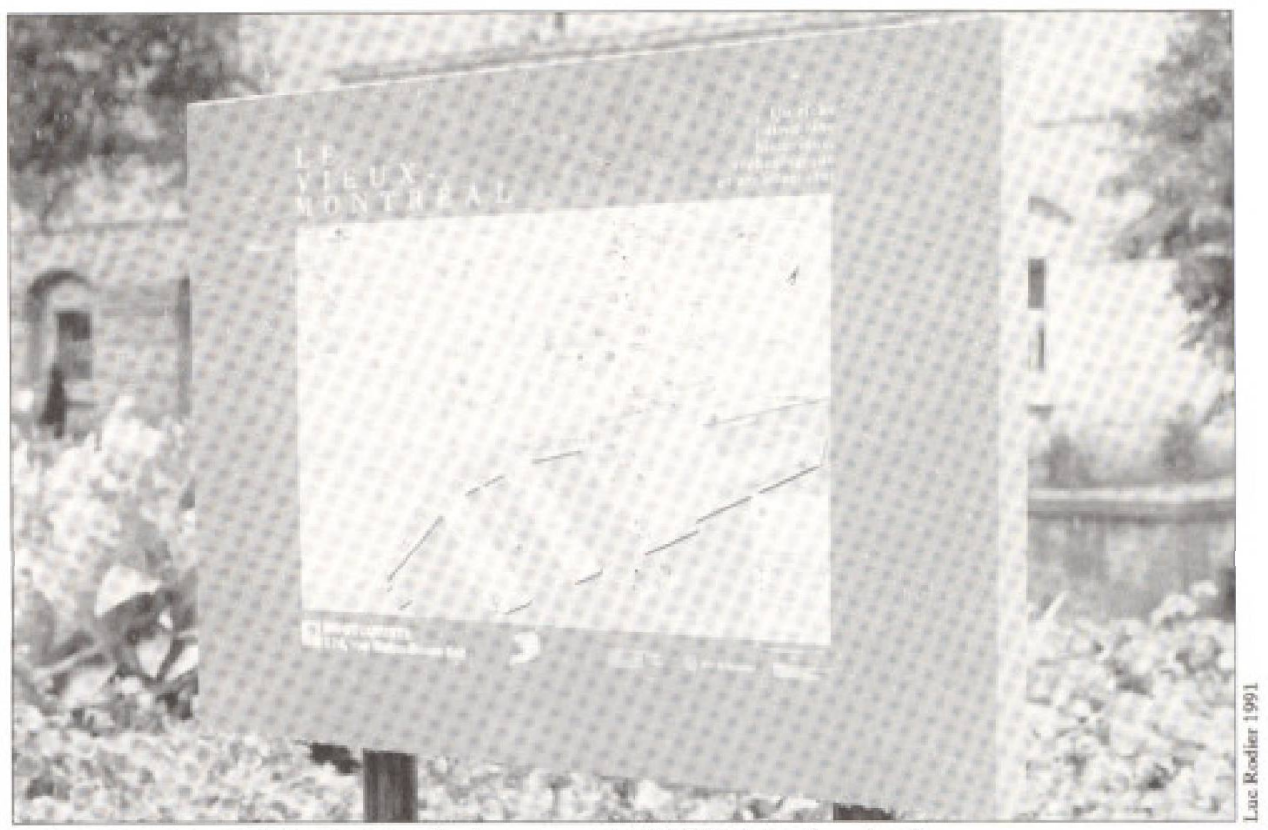

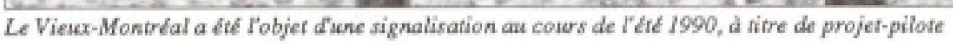

Ce concept est défini comme tel du fait qu'il soriente initialement vers une masse critique touristique (la concentration d'attraits) pour subséquemment orienter vers les attraits spécifiques retrouvés à l'intérieur de la concentration's. Ils'agit, en fait, de diriger le touriste vers un lieu choisi en fonction de la cartographie des localisations des divers attraits touristiques existants et qu'une fois dans cette zone géographique, de l'information spécifique sur des équipements ou activités lui soit donnée pour identifier son lieu de destination finale. Les critères qui ont servi de prémisses au choix de ce type de système sont principalement des critères d'adaptabilité en regard des tracés et du découpage qui sont relativement stables. Il s'agit aussi de critères de flexibilité et de l'adaptabilité de ce scénario au tissu urbain du fait que «la Ville présente des zones d'attraction denses, mais relativement éloignées les unes des autres. Le lien entre les concentrations est assuré par une macro-signalisation simple, alors que la signalisation interne serait assurée par une micro-signalisation plus exhaustives.

Cinqgrandesconcentrations représenteront donc chacune un pôle d'attraction touristique. Ainsi, on retrouvera les pôles du Vieux-Montréal, du Centre-ville, du Parcdes-Iles, du Mont-Royal/Oratoire et le pôle Maisonneuve, qui regroupent les intervenants autour du Parc Olympique et du Jardin Botanique. Le touriste qui arrive à Montréal sera tout d'abord dirigé vers la concentration de son choix par une signa- lisation de pôle qui lui permettra par la suite d'accéder aux différents attraits contenus dans celui-ci. Le réseau sera complété par une signalisation intersectorielle qui indiquera au visiteur comment cheminerd'un pôle à l'autre.

\section{Un projet pilote dans le Vieux-Montréal}

Aux fins de valider les démarches đéjà entreprises par la Ville de Montréal concernant l'implantation de sa politique de signalisation touristique, l'été 1990 fut désignée afin d'effectuer un test de l'approche proposée. De concert avec le ministère des Affaires culturelles, les autorités de la Ville ont choisi le VieuxMontréal comme site d'implantation du projet pilote de la signalisation touristique routière et piétonne.

La signalisation routière du projet pilote consistaiten 18 panonceaux à lettrage blanc sur fond bleu visant à guider les automobilistes des portes d'entrée de la Communauté urbaine de Montréal (CUM) vers le pôle touristique et les stationnements d'accueil.

La signalisation piếtonne, quant à elle, ếtait constituée de 16 cartes du Vieux-Montréal qui ontété implantées pour faire l'interface entre les différentśs modes d'accèsau VieuxMontréal et les déplacements piétonniers.

Le projet pilote du Vieux-Montréal a fait l'objetd'uneétude d'évaluation parl'équipe 
du professeur et expert en la matière, monsieur Romédi Passini, de la Faculté d'amenagement de l'Université de Montréal(5). Pour mieux comprendre en quoi l'implication de ce groupe étail vivement souhaitable, il faut voir que les travaux et recherches réalisés au cours des années par les membres de l'équipe Passini, s'inscrivent dans le champ d'étude des rélations personnes/environnement. Ce champ d'etude rassemble les travaux qui

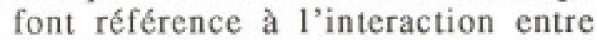
I'individu, ses dimensions physiques et psychologiques et l'aménagement, soit; l'espace bảti et aménagé. La signalisation touristique fait partie intégrantedu domaine de l'orientation spatiale et s'inscrit aussi dans le cadre des phénomènes du champ d'étude personne/environnement sur lequel les chercheurs, sous la gouverne du professeur Passini, se penchent depuis plusieurs années.

Les principales conclusions de l'évaluation du projet pilote précisent entre autres que «la signalisation touristique doit être structuréce de manière encore plus rigoureuse afin que chaque signe s'imbrique avec les autres pour former une suite cohérente d'informations qui mène le touriste vers une destination précises. Quant à l'evaluation de la composante piétonne du projet pilote, l'étude démontre que ales cartes touristiques du projet pilote sont passablement utilisées par les visiteurs, ce qui démontre le bien-fondé de foumir ce type d'information aux visiteurs du VieuxMontréal. De même, l'information livrée par les cartes paraît relativement appréciée par les usagers bien que certaines améliorations s"imposent pour assurer une meilleure continuation $\%$

\section{Une consultation pour les intervenants montréalais}

Lors des nombreuses présentations faites auprès du Comité exécutif, relativement au dossier de la signalisation touristique, l'un des principes directeurs qui avait alors été retenu voulait que le milieu touristique soit appelé à désigner et nommer les pôles touristiques, et ce, sous l'égide de 1'OCTGM. Celle-ci agirait, dans ce cas, comme représentante de la Ville. L'Office organisait, à ce titre, une consultation au mois de mai 1990, avec pour objectif essentiel de connaiture l'opinion du milieu touristique montréalais quant au projet de signalisation touristique.
La proposition initiale présentée aux intervenants, par l'Office des congrès et du tourisme du Grand Montréal, découlait du principe de base qui sous-tend la politique de signalisation, soit la délimitation des pôles touristiques suivant les concentrations d'attraits et $1^{\text {'on }}$ proposait, à cet 6 gard, les cinq pôles à l'étude. Un rapide consensus est intervenu sur le principe de signalisation par concentration d'attraits à partir des autoroutes urbaines. Pendant la consultation, seule des modifications mineures ont été demandées soit en ce qui a trait aux limites de pôles ou à leur dénomination.

\section{Les plans directeurs de signalisation}

Afin de rendre la proposition de système de signalisation opérationnelle, la Ville demandait à quatre firmes de consultants de lui préparer des plans et devis pour toutes les composantes du projet.

La CIDEM et le Service de l'habitation et du développement urbain recevait des firmes désignées, ce qu'il est convenu d'appeler maintenant les plans directeurs routier et piétonnier, les critères de sélection pour la signalisation de proximité et les formats cartographiques ainsi qu'une étude de faisabilité de l'ensemble du projet.

\section{Qu'en est-il de ce système?}

Le système de signalisation touristique repose essentiellement sur des composantes interactives quiont pour principale fonction de guider le touriste dans tous ses déplacements. Au niveau routier, le touriste est d'abord prisen charge dès qu' il franchit l'une des portes d'entrée du territoire de la Communauté́ urbaine de Montréal (CUM) et est dirigé via un réseau d'artères structurantes (réseau d"accueil) de façon prioritaire vers le centre Infotouriste où il pourra trouver toute l'information nécessaire et décider de la poursuite de sa visite de la métropole.

Dans un deuxième temps, un autre réseau d'artères, cette fois de prestige, dit du système intersectoriel, dirige le visiteur vers l'un ou l'autre des cinq pôles touristiques. Cesystême fait appel aux déplacements par automobile mais aussi aux deplacements a pied, par le métro, les circuits d'autobus et le réseau piétonnier intérieur de Montréal.

A l'intérieur dupôle touristique, on offre au touriste une signalisation de proximité qui lui permet de repérer les attraits majeurs du secteur. Ces attraits seront choisis en fonction d'une grille de critères qui vise à identifier les lieux recherchés par les touristes.

Dès que le visiteur a trouvế l'attrait de son choix, on l'invite à laisser sa voiture dans un stationnement $\mathrm{d}^{*}$ accueil d'où il pourra etre pris en charge par une signalisation piétonne. Celle-ci sera essentiellement constitué de supports d'orientation touristiqueetd'information publique sur lesquels on pourra retrouver des cartes du pôle visité. De même, la cohérence du systène piétonnier sera assurée par la composante carte qui sera retrouvéc a l'intérieur des guides touristiques officiels et qui pourrait être reproduite, pour tout autre usage, par les intervenants du pôle.

\section{Conclusion}

Un projet de ceute nature demande plusieurs annés de planification, de consultation et d'opérationnalisation afin de juxtaposer I'ensemble des composantes d'un tel système. La signalisation touristique à Montréal s'est toujours faite en vase clos du fait que chacun des dossiers se traitait à la piece. Obtenir l'appui de l'ensemble des intervenants, pour rassembler les efforts ponctuels de chacun en un seul, ne fut pas une mince affaire. Cependant, pour le bếnéfice du touriste, il est clair qu'un système de signalisation unique et intégré ne pourra qu'améliorer la qualité de l'accueil des visiteurs qui séjournent à Montrấal.

Montréal ne compte d'ailleurs pas s' arrêter à 1992 en ce qui concerne le dossier de la signalisation touristique puisque, d'une part, l'ensemble du projet ci-avant présenté demandera des réajustements et que, de plus, d'autres améliorations sont déjầ sur les planches à dessin.

\section{Notes et références}

(1) Stanley Tremblay, wL'acoueil touristique: le roble de l'Etats, Teoros, vol. 10, no 2, juillet 1991.

(2) SEROM Marketing, Élaboration d'un scenario d'implantation des systames de signalisation touristique pour l'arrondissement Centre de Montréal, octobre 1999.

(3) Romedi Passini, Guylaine Proulx et Sylvain Theriault, Evaluationdu projet piloto de signalisation routière et pibtonne du Vieux-Montrasl, novembre 1990 